\title{
Probiotic and Prebiotic Interventions for Obesity and Diabetes
}

\author{
Rakhie Narayanan, Sreelekshmi K, Honey Chandran C and Keerthi TR* \\ School of Biosciences, Mahatma Gandhi University, Kerala, India
}

ISSN: 2578-0263

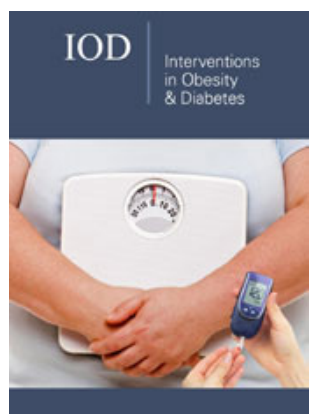

*Corresponding author: Keerthi TR, Professor, School of Biosciences, Kerala, India

Submission: 制July 29, 2019

Published: 制August 12,2019

Volume 3 - Issue 2

How to cite this article: Rakhie $\mathrm{N}$ Sreelekshmi K, Honey C C, Keerthi T. Probiotic and Prebiotic Interventions for Obesity and Diabetes. Interventions Obes Diabetes. 3(2). IOD.000556.2019.

DOI: 10.31031/IOD.2019.03.000556

Copyright@ Keerthi TR, Ning Xu. This article is distributed under the terms of the Creative Commons Attribution 4.0 International License, which permits unrestricted use and redistribution provided that the original author and source are credited.

\begin{abstract}
Gut is considered as second brain since it coordinates with all the organs. A physiological change or dysbiosis in the gut affects the body metabolism. A probiotic treatment along with a restricted diet and an appropriate prebiotic can modulate the host functioning. The mechanisms through which the probiotics improves type 1 , type 2 and gestational diabetes is by modulating insulin resistance, barrier function of gut epithelium, immunomodulation, reducing appetite and upregulating genes for fatty acid breakdown. Fermentation of prebiotics in the gut produces short chain fatty acids which in turn regulates the metabolic pathways by interacting with various receptors and modulating the downstream pathways. The studies reported till date reveals that a synbiotic therapy can be an alternative as well as preventive medicine for lifestyle diseases especially diabetes and obesity.
\end{abstract}

Keywords: Obesity; Type 1 diabetes; Type 2 diabetes; Probiotics and prebiotics; Gut microbiota, Diet

Abbreviations: T1D: Type 1 Diabetes; T2D: Type 2 Diabetes; LPS: Lipopolysaccharides; TLR: Toll Like Receptor; GLP: Glucagon Like Peptide; PAMP: Pathogen Associated Molecular Patterns; GPR: G Protein Coupled Receptor; GLUT: Glucose Transporter; BMI: Body Mass Index; VFA: Visceral Fat Area; SCFA: Short Chain Fatty Acid, ITF: Inulin Type Fructan

\section{Introduction}

Diabetes and obesity are two trending metabolic disorder which leads to major complications in all cases despite of age. Both are strictly correlated and hence called diabesity. Diet is the major factor which contributes to diabesity. The microbial diversity and number in the gut environment mainly depends on the food consumed in healthy individuals. The microflora in gut profoundly affects the host metabolism through several mechanisms. There is a significant difference between the microfloral composition in diabetic and nondiabetic people as well as obese and non-obese. High fat/carbohydrate diet always promotes the establishment of putrefying and opportunistic pathogens. Supplementation of probiotics and prebiotics is a good practice to maintain a healthier gut thereby improving the host functions. The review mainly discuss about the beneficial effects of gut flora/probiotics along with prebiotics in T1D, T2D and obesity.

\section{Discussion}

Human gut harbours predominantly four phyla of bacteria Proteobacteria, Firmicutes such as Ruminococcus, Lactobacillus and Clostridium species, Actinobacteria and Bacteroidetes (Bacteroidaceae and Prevotellaceae). Several studies suggest the association between a dysbiosis gut with obesity and diabetes. Obesity and diabetes are clearly associated with a marked reduction in Firmicutes and higher proportion of Bacteroidetes and Proteobacteria. Studies in animal models showed that gut microorganisms induce diet mediated obesity by harvesting more energy from the diet and the body store them as triglycerides leading to T2D [1]. The mechanism underlying diabesity in dysbiosis includes alteration in fatty acid metabolism, modulation in gut peptide YY and GLP -1 secretion, activation of TLRs by bacterial LPS which elicits immune response thereby modulating the membrane permeability and causes insulin resistance [2]. Same mechanisms were observed in T1D children, but they are generally non obese. Their fecal microbiota of is less diverse and stable [3]. Further the PAMPs mediated inflammation lead to the destruction of beta cells of islets of langherhans thus predispose the pathogenesis of T1D [4]. A healthy and balance gut microbe along with a more vegetarian diet can improve or delay the chronic effects of both type 1 and type 2 diabetes. It also helps to maintain the glucose and fat metabolism as well as alleviates the inflammatory responses. Species of Lactobacillus and Bifidobacteria are extensively explored for their health promoting effects and they are considered as probiotics. According to WHO/ 
FAO "Probiotics are live microorganisms which when administrated in adequate amounts confer health benefits to the host". Probiotics have a characteristic of producing SCFAs from dietary fibers. SCFAs especially butyrate and propionate activate GPR 41 and GPR 43 which further activates GLP-1 and PYY which inhibit gut motility and reduce appetite thus reducing the energy uptake. Butyrate and Propionate also activates gluconeogenesis through cAMP dependent pathway and also decreases the permeability of intestinal barrier [5]. A metagenomic approach in T2D patients revealed a marked decrease in butyrate producing bacteria and an increase in opportunistic pathogens [6].

The antiobesity effect of Lactobacillus gasseri is well known. It reduces the adiposite tissue weight in high sucrose fed rat and reduce glucose level in T2D mice. In mice the same strain significantly increased the mRNA levels of fatty acid oxidation and lowers the expression of genes involved in synthesis of fatty acids and also elevated the glucose transporter GLUT-4 [7]. Probiotic supplementation with low calorie diet can reduce the BMI in adults. A 12-week administration of L. gasseri SBT2055 was enough to reduce BMI, hip circumference and abdominal VFA in Japanese adults [8]. The strain Lactobacillus plantarum reduces the subcutaneous and mesenteric fat mass in high fat fed mice [9] The probiotic fermented soya and flaxseed milk increased insulin production and thereby reduced blood glucose level in alloxan induced T1D rat [10]. Administration of Bifidobacterium breve reduces the accumulation of epididymal fat in a dose dependent manner [11]. L. acidophilus, L. casei, B. lactis, S. thermophilus and E. faecium are other probiotics with antiobesity activity. L. acidophilus and $B$. lactis in yogurt reduced fasting blood sugar,total cholesterol and low-density lipoprotein in T2D patients [12]. Administration of probiotic L. rhamnosus GG and B. lactis in pregnant women from early pregnancy helps in reduction of weight and waist circumference after postpartum [3]. Another study reported a reduction in chance of gestational diabetes mellitus [13]. Probiotics can exert their effect only when they colonize the gut. The nondietary non digestible components in food can be fermented and utilized by probiotics as energy sources. These components are collectively called prebiotics. It is defined by FAO/WHO as "Non digestible food ingredients that beneficially affect the host by selectively stimulating the growth and activity of one or a limited number of bacterial species already established in the colon, and thus improve the host health [14]. Since they are non-digestible, reaches the distal part of GI tract where the resident bacteria utilize it as nutrients. Galacto oligosaccharides, Fructo oligosaccharides, Soybean oligosaccharides, Inulin, Coclodextrins, Gluco oligosaccharides, Xylo oligosaccharides, Lactulose, Lactosucrose and Isomalto oligosaccharides are commonly used for the enriched growth and establishment of probiotics [15]. A study in obese women treated with ITF showed a selective change in gut flora that is increased Bifidobacterium and Faecalibacterium prausnitzii which have a negative impact on serum LPS, fat mass and plasma phosphatidyl choline levels [16]. Arabinoxylan oligosaccharide from wheat bran improves the physiological and metabolic impairments caused by high fat diet in obese mice. It reduces the high fat induced hyperinsulinemia, metabolic endotoxemia, weight gain and fat mass also upregulated the alterations in tight junction [17]. The synergistic form of prebiotic and probiotic called synbiotic selectively and effectively modulate specific microbes of the gut. Dietary ITF consumption selectively modulated Bifidobacterium spp. and decreased fecal SCFA levels in obese women [18]. FOS supplementation in healthy individuals promote satiety, reduce hunger and food ingestion [19] whereas FOS administration in obese resulted in weight loss [20]. In high fed mice FOS restored Bifidobacterium sp. numbers and normalize the LPS level, glucose tolerance and insulin secretion [21]. Antiobesogenic effect of cyclodextrins can be explained by increased level of lactic acid and SCFA in obese mice through the expression of genes involved in lipid metabolism (PPAR and PPAR $\gamma$ ) [22]. Likewise, obese rat fed with prebiotic insulin and oligofructose showed elevated level of circulating GLP-1 and enhanced expression of pro-glucagon and Peptide YY genes [23]. The synbiotic effect of Lactobacillus acidophilus ATCC 4962, fructo-oligosaccharide, inulin, and mannitol in hypercholestremia was showed in high fat fed pigs. It was observed that total cholesterol, low density lipoprotein and triglycerides were reduced [24]. The synbiotic Bifidobacterium animalis subsp. lactis BB-12 and oligofructose improved glycemic condition in obese rat [25]. Daily administration of insulin or maltodextrose in T2D women modulated inflammation and metabolic endotoxemia [26]. Recent studies explain that the enteroendocrine peptides like GLP-1, PYY, and ghrelin which plays important role in glucose homeostasis can be modulated by inulin [27]. ITF can stimulate GLP-1 and GLP-2 synthesis by increasing endocrine L cells in rhodents. GLP-1 reduce appetite, fat mass and insulin resistance and GLP-2 decreases the permeability of intestine and reduce endotoxemia related to diabesity [28]. A four-week administration of FOS reduces hepatic glucose synthesis whereas in T2D patients, oligofructose showed a significant decrease in blood glucose after 6 weeks of administration [29]. The decrease in intestinal low-grade inflammation, correlated with improved gut barrier integrity and reduced proinflammatory cytokines levels, provides an improvement in glucose tolerance and insulin sensitivity [21]. All these findings highlight the use of prebiotics in improving glycemic control and diabetes.

\section{Conclusion}

Invivo studies in animals and humans proved the importance of maintaining a healthy gut in metabolism and homeostasis. Supplementation of probiotics and prebiotics improve the health of the host. A single probiotic cannot satisfy all the health requirements rather they can be used as personalized or occupation-based medicine. Synbiotic therapy is a promising strategy for lifestyle disease like obesity and diabetes. It can prevent the pathogenesis and to some extent can cure these types of metabolic dysfunctions. However more human trails are required to extensively use probiotics in medical practice.

\section{Acknowledgement}

The authors hereby acknowledge Department of Science and Technology, Govt. of India funded under PURSE phase II and FIST programme and Kerala State Council for Science Technology and 
Environment, State Govt. of Kerala for funding and providing infrastructure.

\section{References}

1. Anon (2010) Obesity, diabetes, and gut microbiota. 33(10).

2. Musso G, Gambino R, Cassader M (2010) Obesity, diabetes, and gut microbiota: The hygiene hypothesis expanded? Diabetes Care 33(10): 2277-2284

3. Giongo A, Gano KA, Crabb DB, Mukherjee N, Novelo LL, et al. (2011) Toward defining the autoimmune microbiome for type 1 diabetes. ISME Journal 5(1): 82-91.

4. Neil Munro (2016) Gut microbiota: Its role in diabetes and obesity. Diabetes \& Primary Care 18(4): 1-6.

5. Harsch IA, Konturek PC (2018) The role of gut microbiota in obesity and type 2 and type 1 diabetes mellitus: New insights into 'old' diseases. Medical Sciences 6(2): E32.

6. Wang Jun, Junjie Qin, Yingrui Li, Zhiming Cai, Jianfeng Zh, et al. (2012) A metagenome-wide association study of gut microbiota in type 2 diabetes. Nature 490(7418): 55-60.

7. Kang JH, Yun SI, Park MH, Park JH, Jeong SY, et al. (2013) Anti-obesity effect of lactobacillus gasseri BNR17 in high-sucrose diet-induced obese mice. PLoS ONE 8(1): 1-8.

8. Kadooka Y, Sato M, Imaizumi K, Ogawa A, Ikuyama K, et al. (2010) Regulation of abdominal adiposity by probiotics (lactobacillus gasseri SBT2055) in adults with obese tendencies in a randomized controlled trial. European Journal of Clinical Nutrition 64(6): 636-643.

9. Kim S, Sang DL (2017) Physiological characteristics and anti-obesity effect of lactobacillus plantarum K6 isolated from kimchi. Journal of Milk Science and Biotechnology 35(4): 221-231.

10. Soundharya K, Veerichetty V, Saraswathy N, Balaji S, Ramalingam $P$ (2018) Antidiabetic potential of the combination of fermented soymilk and flaxseed milk in alloxan-induced diabetic rats. International Journal of Green Pharmacy (IJGP) 12(03): 7-8.

11. Kondo S, Xiao JZ, Satoh T, Odamaki T, Takahashi S, et al. (2010) Antiobesity effects of bifidobacterium breve strain b-3 supplementation in a mouse model with high-fat diet-induced obesity. Bioscience, Biotechnology, and Biochemistry 74(8): 1656-1661.

12. Ejtahed HS, Mohtadi NJ, Homayouni RA, Niafar M, Asghari JM, et al. (2011) Effect of probiotic yogurt containing lactobacillus acidophilus and bifidobacterium lactis on lipid profile in individuals with type 2 diabetes mellitus. Journal of Dairy Science 94(7): 3288-3294.

13. Luoto R, Laitinen K, Nermes M, Isolauri E (2010) Impact of maternal probiotic-supplemented dietary counselling on pregnancy outcome and prenatal and postnatal growth: A double-blind, placebo-controlled study. British Journal of Nutrition 103(12): 1792-1799.

14. Pineiro M, Asp NG, Reid G, Macfarlane S, Morelli L, et al. (2008) FAO technical meeting on prebiotics. J Clin Gastroenterol 3:156-159.
15. Younis K, Ahmad S, Jahan K (2015) Health Benefits and Application of Prebiotics in Foods. Journal of Food Processing \& Technology 6(4).

16. Dewulf EM, Cani PD, Claus SP, Fuentes S, Puylaert PG, et al. (2013) Insight into the prebiotic concept: Lessons from an exploratory, double blind intervention study with inulin-type fructans in obese women. Gut 62(8): 1112-1121.

17. Neyrinck AM, Van Hée VF, Piront N, De Backer F, Toussaint O, et al. (2012) Wheat-derived arabinoxylan oligosaccharides with prebiotic effect increase satietogenic gut peptides and reduce metabolic endotoxemia in diet-induced obese mice. Nutrition and Diabetes 2: e28.

18. Salazar N, Dewulf EM, Neyrinck AM, Bindels LB, Cani PD, et al. (2014) Inulin-type fructans modulate intestinal bi fi dobacterium species populations and decrease fecal short-chain fatty acids in obese women. Clin Nutr 34(3): 501-507.

19. Cani PD, Joly E, Horsmans Y, Delzenne NM (2006) Oligofructose promotes satiety in healthy human : A pilot study. Eur J Clin Nutr 60(5): 567-572.

20. Parnell JA, Reimer RA (2013) Weight loss during oligofructose supplementation is associated with decreased ghrelin and increased peptide YY in overweight and obese adults. Am J Clin Nutr 89(6): 17511759.

21. Cani PD, Neyrinck AM, Fava F, Knauf C, Burcelin RG, et al. (2007) Selective increases of bifidobacteria in gut microflora improve high-fatdiet-induced diabetes in mice through a mechanism associated with endotoxaemia. Diabetologia 50(11): 2374-2383.

22. Karaki S, Mitsui R, Hayashi H, Kato I, Sugiya H, et al. (2006) Short-chain fatty acid receptor, GPR43, is expressed by enteroendocrine cells and mucosal mast cells in rat intestine. Cell Tissue Res 324(3): 353-360.

23. Cani PD (2005) On glycaemia-lowering effect of oligofructose in streptozotocin-treated rats. 1: 457-465.

24. Cani PD, Possemiers S, Van de WT, Guiot Y, Everard A, et al. (2009) Changes in gut microbiota control inflammation in obese mice through a mechanism involving GLP-2- driven improvement of gut permeability. Gut 58(8): 1091-1103.

25. Gibson GL, Marcel BR, Catholique DL (1995) Critical review dietary modulation of the human colonie microbiota : Introducing the concept of prebiotics.

26. Dehghan P, Gargari BP, Jafar-Abadi MA, Aliasgharzadeh A (2014) Inulin controls inflammation and metabolic endotoxemia in women with type 2 diabetes mellitus: A randomized-controlled clinical trial. Int J Food Sci Nutr 7486(1): 117-123.

27. Cani PD, Everard A, Duparc T (2013) Gut Microbiota, Enteroendocrine Functions and Metabolism. Current Opinion in Pharmacology 13(6): 935-940.

28. Cani PD, Dewever C, Delzenne NM (2004) Inulin-type fructans modulate gastrointestinal peptides involved in appetite regulation (glucagon-like peptide-1 and ghrelin) in rats . Br J Nutr 92(3): 521-526.

29. Cicek B, Arslan P, Kelestimur F (2009) The effects of oligofructose and polydextrose on metabolic control parameters in type-2 diabetes. Professional Medical Publications 25(4).

For possible submissions Click below: 